\title{
Study on the Dose-effect Relationship of Moxa Burning Fumigation in Air Disinfection in Traditional Chinese Medicine Clinics
}

\author{
Shunhua Teng*, Guoyong Ma, Guozhong Lyu \\ Scientific Research Room, Yuzhong County Hospital of Traditional Chinese Medicine, Lanzhou 730100, Gansu, China \\ Email: 1421449910@qq.com
}

\begin{abstract}
Objective - It's to study the dose-effect relationship of moxa produced by Gansu Baicao Group in the air disinfection in traditional Chinese medicine clinics. Method - Use moxa sticks burning fumigation to disinfect indoor air in the clinics of traditional Chinese medicine experts; use the natural sedimentation method to detect the bacterial content in the air; compare the sterilization effects of $0.5,1$ and 1.5 moxa sticks $0 \mathrm{~h}, 1 \mathrm{~h}$ and $2 \mathrm{~h}$ after air disinfection. Results - In the $54 \mathrm{~m} 3 \mathrm{TCM}$ clinic, there was no significant difference in the sterilization rate of air bacteria $0 \mathrm{~h}, 1 \mathrm{~h}$, and $2 \mathrm{~h}$ after using 1 moxa stick and 1.5 moxa sticks respectively for fumigation and disinfection $(\mathrm{P}>0.05)$. There was a significant difference in the sterilization rate of air bacteria between 1 moxa stick group and 0.5 moxa stick group $0 \mathrm{~h}$ after air disinfection $(\mathrm{P}<0$. 05). There was a significant difference in the sterilization rate of air bacteria between 0 hour and 1 hour after burning fumigation for air disinfection in 0.5 moxa group $(\mathrm{P}<0.05)$. Conclusion - The best dose of moxa burning fumigation for air disinfection in $54 \mathrm{~m} 3$ Chinese medicine clinic is 0.5 stick; the best time for air disinfection is $1 \mathrm{~h}$ after disinfection.

Keywords: traditional Chinese medicine clinic, moxa burning fumigation, disinfection dosage, disinfection time, sterilization effect
\end{abstract}

\section{Introduction}

Air is the transmission medium of many diseases and an important way for pathogenic microorganisms to spread. How to carry out effective air disinfection and reduce infections caused by air pollution has always been an important topic of concern to the medical community. The hospital is a densely populated place with more pathogenic microorganisms floating in the air, which directly causes potential harm to patients and medical staff. Therefore, air disinfection is an important measure to prevent epidemics and prevent cross-infection in the hospital. Modern studies have confirmed that moxa fumigation has the advantages of bacteriostasis and antivirus in terms of air disinfection. However, in the air disinfection technology of moxa burning fumigation, if the burning dose and disinfection time of moxa sticks are not well mastered, it will directly affect the disinfection effect. Therefore, in order to explore the best disinfection dose and time of moxa burning fumigation air disinfection, we used moxa stick burning fumigation method to disinfect the air in the clinics of traditional Chinese medicine experts from June 2020 to November 2020. Disinfection time and effect were analyzed. The process and results are described as follows.

\section{Materials and methods}

\subsection{Materials}

HN-40S electric heating constant temperature incubator produced by Shanghai Lichen Bangxi Instrument Technology Co., Ltd.; 90mm blood agar plate produced by Zhengzhou Antu Technology Co., Ltd.; Moxa sticks produced by Gansu Baicao Group, $20 \mathrm{~cm}$ long, $1.8 \mathrm{~cm}$ in diameter, and $28 \mathrm{~g}$ in weight.

The experimental site is the clinics of Chinese medicine experts. Four clinics with the same volume $(3 \mathrm{~m} \times 6 \mathrm{~m} \times$ $3 \mathrm{~m}$ ) and the same indoor structure (doors, windows, indoor facilities) were selected as disinfection objects, and they were randomly selected during the experiment.

\subsection{Experimental methods}

\subsubsection{Experiment groups}

According to the dose of moxa burning fumigation and disinfection, the experiment was divided into 3 groups; group A: 0.5 moxa stick; group B: 1 moxa stick; group C: 1.5 moxa sticks.

\subsubsection{Moxa burning method}

Close doors and windows during disinfection to reduce air circulation and personnel movement. Insert $0.5,1$ and 1.5 
moxa sticks produced by Gansu Baicao Group into the moxibustion small plate hole produced by Gansu Baicao Group, ignite both ends, and burn for 1 hour according to the experimental requirements. Moxa burning air was disinfected and sampled at 2 o'clock in the afternoon every day, 30 cases were sampled in each group. (Note: The smoke detector will automatically alarm when the dose of moxa stick exceeds $42 \mathrm{~g}$ for 1 hour).

\subsubsection{Gravity plate method}

Samples were taken for each moxa group without disinfection and $0 \mathrm{~h}, 1 \mathrm{~h}$ and $2 \mathrm{~h}$ groups after disinfection. According to the sampling requirements, place a blood agar plate with a diameter of $90 \mathrm{~mm}$ in a fixed position (select 3 points from the wall $\mathrm{lm}$ at the midpoint and both ends of the diagonal); place it horizontally at a height of $\mathrm{lm}$ from the ground and expose it for 5 minutes Cover it; send it to the culture room for 48 hours in a $35^{\circ} \mathrm{C}$ incubator; calculate the average number of colonies in 3 petri dishes.

\subsubsection{Observation indicators}

The total number of air bacteria $\left(\mathrm{cfu} / \mathrm{m}^{3}\right)=50000 \mathrm{~N} / \mathrm{AT}[\mathrm{N}$ is the number of bacterial colonies, A is the area of the plate $\left(\mathrm{cm}^{2}\right), \mathrm{T}$ is the plate exposure time (min)], calculate the number of colonies in the air $\left(\mathrm{cfu} / \mathrm{m}^{3}\right)$.

Bacteria sterilization rate $=[$ (number of bacteria before disinfection - number of bacteria after disinfection $) /$ number of bacteria before disinfection] $\times 100 \%$.

The total number of air bacteria $\left(\mathrm{cfu} / \mathrm{m}^{3}\right)=50000 \mathrm{~N} / \mathrm{AT}[\mathrm{N}$ is the number of bacterial colonies; A is the area of the plate $\left(\mathrm{cm}^{2}\right)$; $\mathrm{T}$ is the plate exposure time (min)]. Calculate the number of colonies in the air $\left(\mathrm{cfu} / \mathrm{m}^{3}\right)$. The criteria for determining the qualified rate of air disinfection are the various environmental air hygiene standards in the "Disinfection Management Measures" promulgated by the Ministry of Health. The number of bacteria in the air in the outpatient clinic of Chinese medicine experts is $\leq 500 \mathrm{cfu} / \mathrm{m}^{3}$, which is qualified for air disinfection.

\subsection{Statistical methods}

The SPSS statistical software package is used for statistics and analysis of the above data. The number of bacteria is expressed by " $\mathrm{x} \pm \mathrm{S}$ "; the sterilization rate is expressed by "\%"; the t value test is used. When the result is $\mathrm{P}<0.05$, it indicates that the comparison is statistically significant.

\section{Results}

In the clinics of Chinese medicine experts, the comparison of the content of bacteria in the air (different disinfection dosage and different disinfection time) after air disinfection by moxa burning fumigation is shown in Table 1 .

Table 1. Comparison of effects of moxa burning fumigation before and after air disinfection (different doses) (measurement result: cfu/ $\mathbf{m}^{3}$ )

\begin{tabular}{|c|c|c|c|c|c|}
\hline \multirow{2}{*}{ Groups } & \multirow{2}{*}{$\begin{array}{l}\text { Number of bacteria before } \\
\text { disinfection }\end{array}$} & \multicolumn{4}{|c|}{ Number of bacteria after disinfection/(sterilization rate \%) } \\
\hline & & & $0 \mathrm{~h}$ & $1 \mathrm{~h}$ & $2 \mathrm{~h}$ \\
\hline A & 520.33 & 88.76 & $(82.94)$ & $21.02(95.96) *$ & $21.12(95.94)$ \\
\hline B & 626.70 & 24.58 & $(96.07) \boldsymbol{\Delta}$ & $24.12(96.15)$ & $24.12(96.15)$ \\
\hline $\mathrm{C}$ & 625.13 & 25.12 & $(95.98) \bullet$ & $20.34(96.75)$ & 20.47 (96.73) \\
\hline
\end{tabular}

Note: * Compare $0 \mathrm{~h}$ and $1 \mathrm{~h}$ after burning fumigation in group $\mathrm{A}, \mathrm{P}<0.05$; compare $1 \mathrm{~h}$ and $2 \mathrm{~h}, \mathrm{P}>0.05$.

$\Delta$ Compare group $\mathrm{A}$ and group $\mathrm{B} 0 \mathrm{~h}$ after burning fumigation, $\mathrm{P}<0.05$.

- Compare group $\mathrm{B}$ and group $\mathrm{C} 0 \mathrm{~h}$ after burning fumigation, $\mathrm{P}>0.05$.

\section{Discussion}

\subsection{The best disinfection dose of moxa burning fumigation air disinfection is 0.5}

It can be seen from Table 1 that after burning and disinfecting in $0.5,1$ and 1.5 moxa sticks groups, the average bacterial content in the air decreased significantly compared with that before disinfection, while in 0.5 moxa stick group, 1 moxa stick group and 1.5 moxa sticks groups, the average bacterial sterilization rate in the air was more than $95 \%$ at 1 $\mathrm{h}$ and $2 \mathrm{~h}$ after air disinfection. The average sterilization rate of air in 1 moxa stick group and 1.5 moxa sticks group was 
more than $95 \%$ at $0 \mathrm{~h}$ after disinfection of fumigated air. All met the air hygiene standards in various environments in the "Disinfection Management Measures" promulgated by the Ministry of Health, and there was no significant difference between groups $(\mathrm{P}>0.05)$. There was no significant difference in the average bacterial sterilization rate in the air between the 1 moxa stick group and the 1.5 moxa sticks group $0 \mathrm{~h}$ after burning air disinfection $(\mathrm{P}>0.05)$. In 0.5 moxa stick group, the average sterilization rate of bacteria in the air was $83 \% 0 \mathrm{~h}$ after disinfection of fumigated air. This does not meet the air sanitation standards in various environments in the "Disinfection Management Measures" promulgated by the Ministry of Health. Compared with the average bacterial sterilization rate of $96.07 \%$ in the air of the moxa stick group, there is a significant difference $(\mathrm{P}<0.01)$. The results show that the optimal disinfection dose of moxa burning fumigation air disinfection is 0.5 .

\subsection{The best air disinfection time for moxa burning fumigation is $1 \mathrm{~h}$ after disinfection}

It can be seen from Table 1 that the average bacterial sterilization rate in the air $0 \mathrm{~h}, 1 \mathrm{~h}$, and $2 \mathrm{~h}$ after air disinfection in the 1 moxa stick group and 1.5 moxa sticks groups is greater than $95 \%$, which is in compliance with the "Disinfection Management" issued by the Ministry of Health The air hygiene standards in various environments in the Measures. In the 0.5 moxa stick group, there was no significant difference in air average bacterial sterilization rate between $1 \mathrm{~h}$ and $2 \mathrm{~h}$ after disinfection $(\mathrm{P}>0.05)$, but there was significant difference between $0 \mathrm{~h}$ after disinfection and $1 \mathrm{~h}$ after disinfection $(\mathrm{P}<$ 0.01). The results show that the best time for air disinfection of moxa burning fumigation is $1 \mathrm{~h}$ after disinfection.

\subsection{The clinical use effect of moxa burning fumigation air disinfection}

Clinical studies have found that ${ }^{[1]}$ Mugwort fumigation has a good effect on air disinfection. The effective ingredients of mugwort fumigation are mainly divided into two parts, one part is the composition of volatile oil, and the other part is the composition of smoke produced by the burning of mugwort. The main component of mugwort is volatile oil, which contains cineole, terpinenol, thujone, camphor, borneol, linalyl, caryophyllene, $\alpha$-phellandrene, camphene and other compounds, in addition to flavonoids, tannin Quality, polysaccharides and other substances ${ }^{[2]}$. Li Yanqiang et al. ${ }^{[3]}$ found that the composition of moxa smoke is different from that of moxa leaves. The volatile components shared by mugwort and mugwort are 1,8-cineole, arthenol, borneol, terpineol, carvol, trans-caryophyllene, large root myrene, caryophyllene oxide, spartol, Chlorophyll alcohol and so on. Among them, 1,8-cineole, arthrol, borneol, and terpineol have higher content in both. The only substances detected in moxa smoke were benzaldehyde, phenol, 2,4-dimethylphenol and melamine. This shows that during the burning process of mugwort, some of the components are pyrolyzed and new substances are produced.

Jin Ran et al. ${ }^{[4]}$ used solid-phase microextraction-gas chromatography-mass spectrometry to qualitatively analyze moxa smoke, and learned that the moxa smoke contains furan structure substances, aromatic compounds, esters, alkanes or hydroxyl-containing compounds. Analyzing the chemical components of mugwort and mugwort, it was found that the antibacterial and anti-inflammatory constituents were mostly eucalyptol, $\beta$-caryophyllene and thujone. The volatile oil of moxa smoke and moxa leaf contains eucalyptol, 2-czone, caryophyllene and its oxides, borneol, etc., which have antibacterial and antiviral effects, and have anti-inflammatory and analgesic effects. In addition, the triterpenoids contained in the wormwood have antibacterial, antiviral, anti-tumor, anti-inflammatory, blood pressure and cholesterol-lowering and immune regulation effects, and are a class of compounds with strong biological activity ${ }^{[5]}$.

Modern research suggests that wormwood fumigation has the advantages of antibacterial and antiviral in air disinfection. Decoction and fumigation of mugwort leaves have a good effect on disinfection and sterilization in hospital wards. A few days ago, the Licha Town government, health center, and nursing homes of Jiaozhou City used moxa moxa to burn and fumigate continuously ${ }^{[6]}$; Zhengzhou City Hospital of Traditional Chinese Medicine also used moxa fumigation to prevent cross-infection caused by COVID-19 ${ }^{[7]}$.

\section{Conclusion}

In summary, the best dose and disinfection time of moxa stick burning fumigation air disinfection in the Chinese medicine clinic is 0.5 stick and 1 hour. This traditional Chinese medicine disinfection method has reached the qualification standards for various environmental air disinfection stipulated by the Ministry of Health. Moxa burning fumigation technology can be used for air disinfection in hospitals.

\section{Acknowledgments}

This article was supported by the following fund project: Gansu Baicao Biotechnology Co., Ltd. Open Fund Project (No.: 2020BC730100). 


\section{References}

[1] Guo Yanling, Liu Yaru. Observation and analysis of the effect of Chinese herbal medicine Atractylodes Rhizome and Artemisia argyi smoke on indoor air disinfection. Chinese Convalescent Medicine. 2010; 19 (3): 259-260.

[2] Gong Jun, Zhang Maomei, Liu Hongwei, et al. Research progress on the chemical constituents and pharmacological effects of mugwort. Guangzhou Chemical Industry. 2018; 46(4): 10-12.

[3] Li Yanqiang, Hu Jun, Zhang Xiaobing, et al. Analysis of Volatile Components of Mugwort and Its Smoke Particles. Tobacco Science \& Technology. 2005; 38(10): 15-17.

[4] Jin Ran, Zhao Baixiao, Yu Mi Mi, et al. Qualitative analysis of moxa combustion product components by solid phase microextraction gas chromatography mass spectrometry. Journal of Beijing University of Chinese Medicine. 2011; 34(9): 632-636

[5] Tian Lu. Study on the chemical composition of mugwort and its anti-inflammatory effect. Guangzhou: Jinan University; 2017.

[6] Cao Sen. Wormwood fumigation to eliminate the epidemic virus, the prevention and control of Chinese medicine in the Licha Town Health Center, Jiaozhou City. Available from: http://qingdao.dzwww.com/xinwen/qingdaonews/202001/t20200128_17258421.htm [Accessed 2020-03-24].

[7] Suo Xiaocan, Zhu Qing. Zhengzhou City Hospital of Traditional Chinese Medicine: The Outpatient Hall is Full of Smoke to Ward Off the Epidemic. Available from: http://bbs1.people.com.cn/post/2/1/2/174664335.html [Accessed 2020-03-24]. 